Chronische Nierenerkrankung erhöht koronares Risiko bei Diabetes

\section{Adäquate Lipidsenkung notwendig}

\author{
Schon lange ist bekannt, dass das kardiovaskuläre Risiko von Patienten bei \\ einer Verschlechterung der Nierenfunktion zunimmt. Dies bestätigen aktuell \\ die Ergebnisse einer großen populationsbezogenen Kohortenstudie [1]. Dys- \\ lipidämien sind bei diesen Patienten weit verbreitet und sollten effektiv be- \\ handelt werden.
}

Auch die vor kurzem aktualisierte Leitlinie der US-amerikanischen National Kidney Foundation und der Kidney Disease Outcomes Quality Initiative (KDOQI) zur Therapie von Diabetes und chronischer Nierenerkrankung (CKD) [2] empfiehlt eine das LDL-Cholesterin senkende Therapie, z.B. mit einem Statin oder einer Ezetimib-Statin-Kombination, um das Risiko schwerwiegender atherosklerotischer Ereignisse bei Patienten mit Diabetes und CKD zu reduzieren. Es handelt sich um eine Empfehlung der Kategorie $1 \mathrm{~B}$.

Mit den Daten von rund 1,3 Mio. Patienten der Datenbank des Alberta Kidney Disease Network (AKDN) und des National Health and Nutrition Survey (NHANES) wurde in einer populationsbasierten Kohortenstudie untersucht, ob eine Niereninsuffizienz ähnlich wie ein Diabetes mellitus als Risikoäquivalent für eine KHK angesehen werden kann. Eine chronische Niereninsuffizienz war definiert als eine errechnete GFR von 15 bis $59,9 \mathrm{ml} / \mathrm{min}$ pro $1,73 \mathrm{~m}^{2}$ (entspr. Stadium 3 oder 4). Primärer Endpunkt war die erste Hospitalisierung wegen eines Herzinfarkts. Während einer medianen Nachbeobachtungszeit von 48 Monaten wurden 11340 von 1268029 Patienten (1\%) wegen eines Herzinfarkts hospitalisiert. Die nicht adjustierte Herzinfarktrate war bei Personen mit vorherigem Infarkt am höchsten (18,5 pro 1000 Personenjahre, 95\%-KI 17,4-19,8). Bei Patienten mit Diabetes und Niereninsuffizienz war die Infarktrate höher als bei Patienten mit nur einer der beiden Erkrankungen. Patienten mit chronischer Niereninsuffizienz ohne früheren Myokardinfarkt hatten signifikant mehr Infarkte als entsprechende Patienten mit Diabetes (6,9 vs. 5,4 pro 1.000 Personenjahre). Es starben mehr Patienten (ohne Diabetes oder bekannte KHK) mit chronischer Nierenerkran- kung (14\%) innerhalb von 30 Tagen nach Hospitalisierung wegen eines Herzinfarkts als Diabetiker $(8 \%, p<0,0001)$ oder jene mit vorangegangenem Infarkt $(10 \%$, $\mathrm{p}=0,0053)$. Eine chronische Nierenerkrankung bedeutet damit für Diabetiker ein sehr hohes Risiko für koronare Ereignisse. Fettstoffwechselstörungen sollten bei dieser Gruppe also adäquat therapiert werden. Diese Empfehlung ist auch in der neuesten Version der Leitlinie der KDOQI zur Therapie von Diabetikern mit chronischer Nierenerkrankung enthalten. Dyslipidämien sind bei diesen Patienten weit verbreitet und kardiovaskuläre Ereignisse sind eine häufige Ursache für Mortalität und Morbidität. Eine Senkung erhöhter LDL-Cholesterinspiegel senkt auch das Risiko für schwerwiegende atherosklerotische Ereignisse. Die Empfehlung für die Ezetimib-StatinKombination basiert auch auf den Ergebnissen der SHARP-Studie (Study of Heart and Renal Protection) [3].

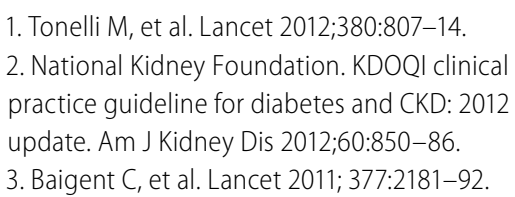

Quelle: Mitteilung des Unternehmens MSD vom 28.2.2013

\section{Diabetes Programm Deutschland: Sie laufen wieder! \\ Die Typ-2-Diabetikerin Karin Schürer war vor ein paar Jahren noch ein Sportmuffel, nun nimmt sie bereits zum dritten Mal am Diabetes Pro- gramm Deutschland teil. Neben vielen begeisterten Läufern mit Diabetes, ist auch das Unternehmen Bayer wieder dabei. „Letztes Jahr hatten alle Beteiligten großen Spaß und Freude an dem gemeinsamen Laufpro- gramm - wir sind als Partner zusammengewachsen", erzählt Claudia Geis, Leiterin Bayer Diabetes Care. Das Unternehmen stattet die Teilnehmer mit Contour XT Blutzuckermessgeräten und Contour Next Sensoren für die Trainingseinheiten und den finalen Lauf aus.}

\section{Bessere Blutzuckerwerte dank Lauftraining}

Laufen gehört zu den beliebtesten und gesündesten Sportarten. Es trainiert das Herz-Kreislaufsystem, wirkt sich positiv auf den Blutzuckerspiegel aus und regt die Produktion von Endorphinen (Glückshormonen) an. Doch aller Anfang ist schwer: Vor allem beim Vorliegen einer Stoffwechselstörung wie Diabetes mellitus sollten Anfänger auf professionelle Hilfe zurückgreifen, denn durch den hohen Energieverbrauch kann schnell eine Unterzuckerung entstehen. Deshalb ist es wichtig, dass Läufer mit Diabetes auf Basis präziser Messwerte gut eingestellt sind. Mit dem Ziel, Menschen mit Diabetes zu mehr Sport zu motivieren, geht das Diabetes Programm Deutschland im Mai in die dritte Runde. Neben regelmäßigen Lauftrainings über einen Zeitraum von sechs Monaten, gibt es eine therapeutische und fachmedizinische Betreuung beispielsweise durch Exper- ten der Sporthochschule Köln sowie der Berliner Charité. Das Ziel ist die Teilnahme von möglichst vielen Läufern mit Diabetes an einem großen Eventlauf in Hamburg, Berlin oder Köln über fünf Kilometer, zehn Kilometer, die Halbmarathon- oder sogar über die Marathon-Distanz. „Wir bieten für jede Ausdauerstufe Laufgruppen an: von laufwilligen Anfängern bin hin zu erfahrenen Marathonläufern", erklärt Initiator und Typ-1-Diabetiker Dr. Michael Rosenbaum.

\section{Beeindruckende Ergebnisse}

Wie Menschen mit Diabetes von regelmäßiger sportlicher Aktivität profitieren, belegen die beeindruckenden Effekte, von denen die Läufer des Diabetes Programm Deutschland berichten. Karin Schürer: „Mein Nüchtern-Wert liegt mittlerweile bei $100 \mathrm{mg} / \mathrm{dl}$. Zudem habe ich 18 Kilogramm abgenommen, seitdem ich beim Laufprogramm mitmache."

Menschen mit Diabetes, die Interesse an der Teilnahme am Laufprogramm haben, können sich anmelden unter www.diabetes-programmdeutschland.de. Unter der kostenfreien Bayer-ServiceHotline (Tel. 0800/50 888 52) stehen rund um die Uhr Experten für Fragen zur Blutzuckerselbstmessung zur Ver-

fügung. Quelle: Bayer Health Care

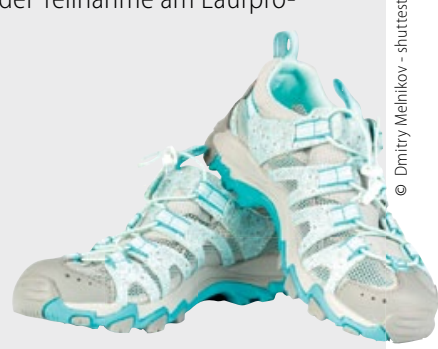

